\title{
Optimal Design of Smart Mobile Terminal Antennas for Wireless Communication and Computing Systems
}

\author{
Junwei Lu and *Shiyou Yang \\ Griffith School of Engineering, Griffith University, Qld 4111, Australia \\ * College of Electrical Engineering, Zhejiang University, Zhejiang, P.R.China
}

\begin{abstract}
The paper presents an electronically beam switched smart mobile terminal antenna (SMTA) array used in wireless network. SMTA arrays were optimized for the secured communication performance and desired radiation coverage. The radiation beam pattern toward desired direction is controlled by switched beam control algorithm. Four different configurations of SMTA arrays and their design considerations are discussed in this paper.
\end{abstract}

Keywords: Smart Mobile Terminal Antenna, Wireless Communications, Wireless Network.

\section{Introduction}

Mobile wireless computing network has become a significant area of growth recently. The technology of smart or adaptive antennas for base station of mobile communications has received enormous interests worldwide in recent years. However, the application of smart antennas for mobile wireless computing terminals and hand-held mobile devices has received little attention due to the smart mobile terminal antenna not being available. The main reason is that the physical size of traditional smart antennas based on linear antenna array with $1 / 2$ wavelength separation in space is too large to fit into any mobile wireless computing terminal and handheld mobile equipment. Therefore, the smart mobile terminal antennas with small size are desperately needed by wireless communications systems. This paper mainly discusses the seven elements based electronically beam switched antenna arrays and its optimal design, and application in wireless communications and computing systems.

\section{Smart Mobile Terminal Antennas (SMTAs)}

Unlike traditional antennas, such as omnidirectional and directional patch antennas applied to current wireless communication and computing systems, the proposed SMTA arrays using switched beam or adaptive beam-forming technologies can significantly increase the security level in wireless network [1]. Figure 1 (a) - (c) illustrates the application of SMTAs in 1-4244-1372-9/07/\$25.00 @2007 IEEE wireless communication and computing systems, where the switched beam only covers the desired direction and area.

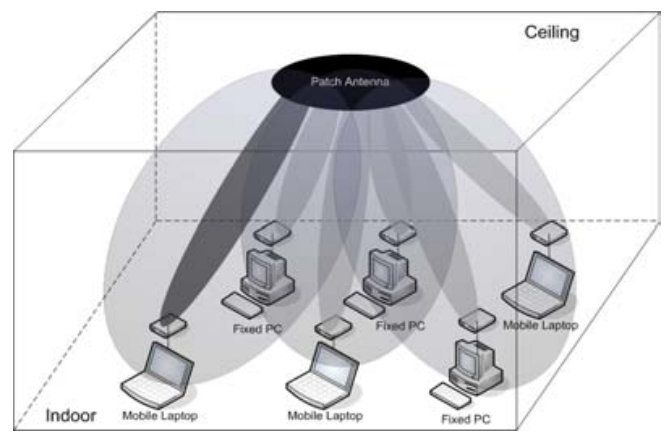

(a)

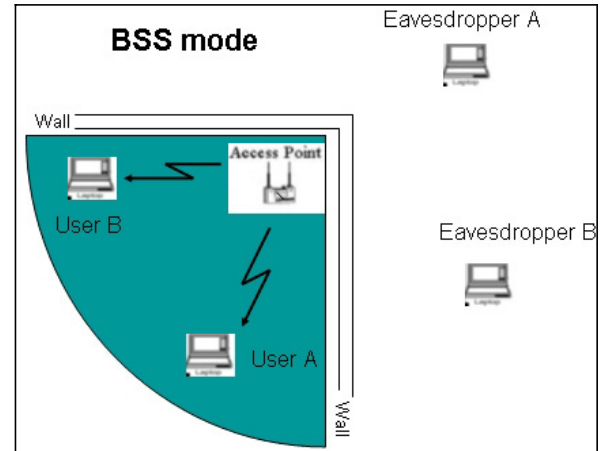

(b)

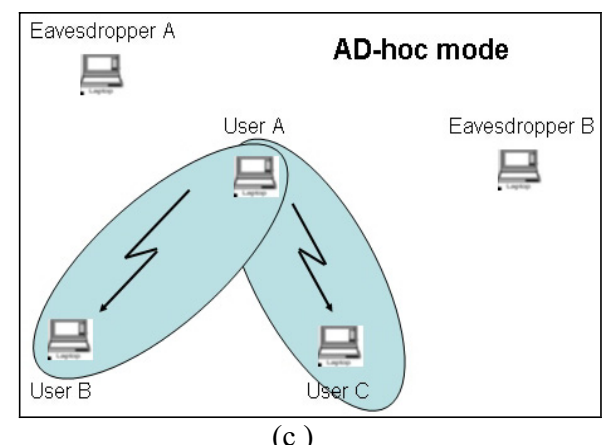

Fig. 1. SMTA used in wireless communication and computing system, (a) Ceiling mounting SMTA, (b) SMTA for Desktop or notebook computers in BSS mode, (c) SMTA for wireless network in Ad-Hoc mode. 


\section{Design Consideration of SMTA}

As mentioned in the above section, the SMTA is not a traditional phase array antenna, all parasitic element working together with source element for a directional beam. Therefore the optimal design is necessary during the antenna design. In this point of view, the antenna configurations as studied in this and the next sections are all optimized by using a GA algorithm with respect to the following goals: the antenna size, reflection coefficient, and beam bandwidth should be as small as possible; and the antenna gain should be as large as possible. Moreover, other considerations about three basic antenna configurations are discussed as follows.

\section{A. Wire SMTA}

Figure 2 shows the configuration of wire SMTA which consists of seven elements, ground plan and skirt. The central element is a driven element (No.0) connected to RF source and other six elements are parasitic elements (No.1-6) connected to beam control circuit. Unlike the traditional linear antenna arrays that have to keep $0.5 \lambda_{0}$ separation, the monopole antenna array based wire SMTA has the element separation around $0.25 \lambda_{0}$ to each other [2]. The other major difference compared with the traditional smart antenna arrays is that a wire SMTA only requires a single RF source.

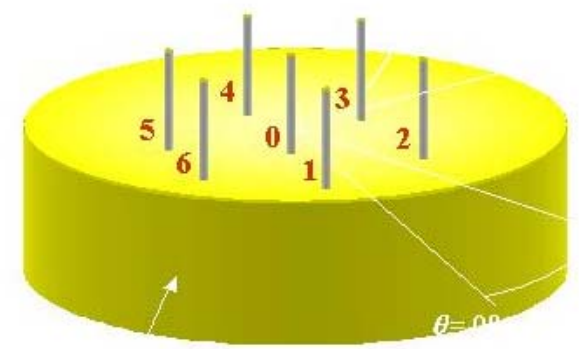

Fig. 2. Configuration of a seven element based wire SMTA.

\section{B. Dielectric Embedded SMTA}

In mobile terminals the size of antenna is an important issue as the portability is always a key requirement. Phased array antennas require an interelement spacing of greater than $0.5 \lambda_{0}$. In wire SMTA, the separation distance can be reduced to $0.25 \lambda_{0}$. Despite this, antennas designed for the IEEE 802.11 standard and Bluetooth at $2.4 \mathrm{GHz}$ are still considered too large to be integrated into the computer and portable devices. If the antenna elements are embedded in the high dielectric medium (non-magnetic material) as shown in Fig. 3, the wavelength will be $\lambda_{r}=\lambda_{0} / \sqrt{\varepsilon_{r}}$ and the reduction factor $F_{r}$ for the height of antenna elements will be expressed as $F_{r}=\sqrt{\varepsilon_{r}}$. Further size reduction based on optimised design techniques was achieved by embedding an array of wire monopoles in a dielectric material [3].

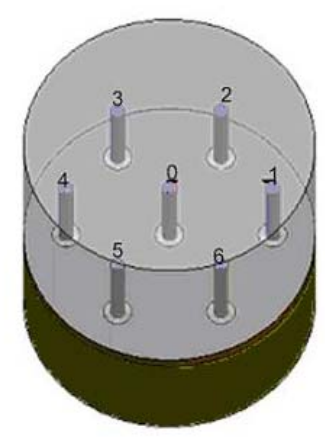

Fig. 3. Seven elements based dielectric embedded SMTA.

\section{Patch SMTA}

The newly developed electronically beam steerable microstrip patch SMTA, as shown in Fig. 4, can be mounted in ceilings for indoor wireless network applications [4]. The central element is fed by two orthogonal feed points with a $90^{\circ}$ phase difference to generate left-hand circular polarization of the current distribution. Thus the beam-steering can be carried out equally through six directions in azimuth. Beamsteering is achieved by either open-circuiting or shortcircuiting the parasitic antenna elements by switches or reactive loads where an open-circuited element acts as director while a short-circuited element effectively becomes transparent. The fields generated by each element couple together to formulate the total far field. Due to the induced circular-polarized currents in the parasitic elements, two short-circuited points are required for each element to effectively terminate any current presence.

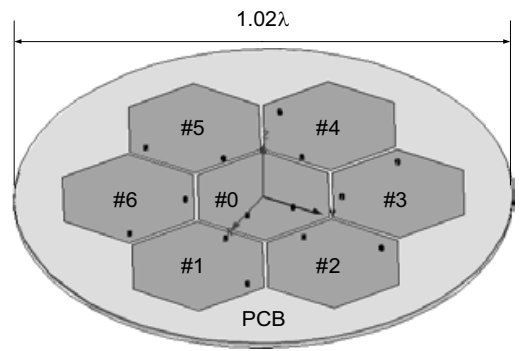

( a ) Hexagonal patch SMTA 


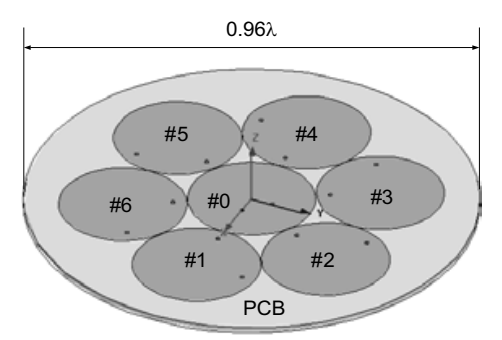

(b) Circular patch SMTA

Fig. 4. Seven elements based Patch SMTAs.

\section{Simulation and Experiment Results}

\section{A. Results of Wire SMTA}

As explained preciously, the Wire SMTA was designed and optimized by using a GA in which the FEM based full wave simulator, HFSS in frequency domain, is used. Figure 5 and Figure 6 show the reflection coefficient and radiation pattern of Wire SMTA at operating frequency respectively.

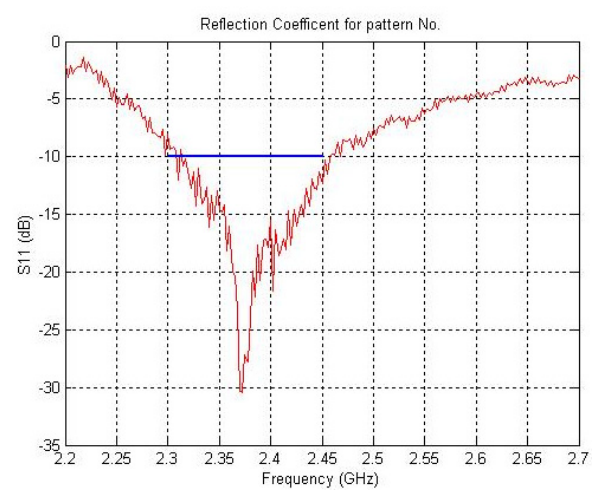

Fig. 5. Measured reflection coefficient $S_{11}$ for a 7-Element Wire SMTA.

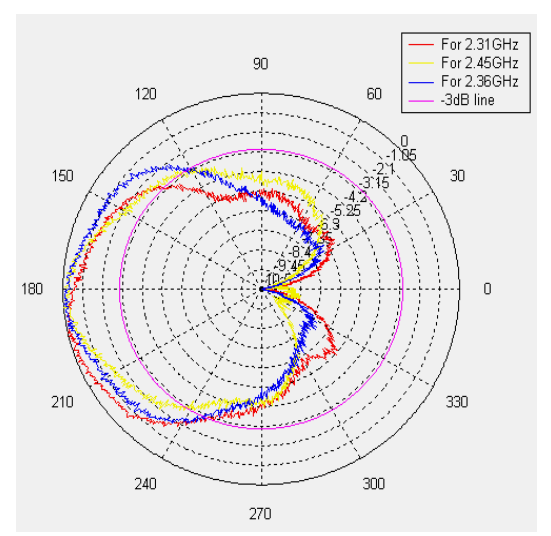

Fig. 6. Measured H-Plane radiation pattern for a 7-Element Wire SMTA at $2.3 \mathrm{GHz}, 2.45 \mathrm{GHz}$ and $2.36 \mathrm{GHz}$.

\section{B. Results of Dielectric Embedded SMTA}

The simulation results compared with measured results for an optimized dielectric embedded SMTA are shown in Fig. 7 and Fig. 8.

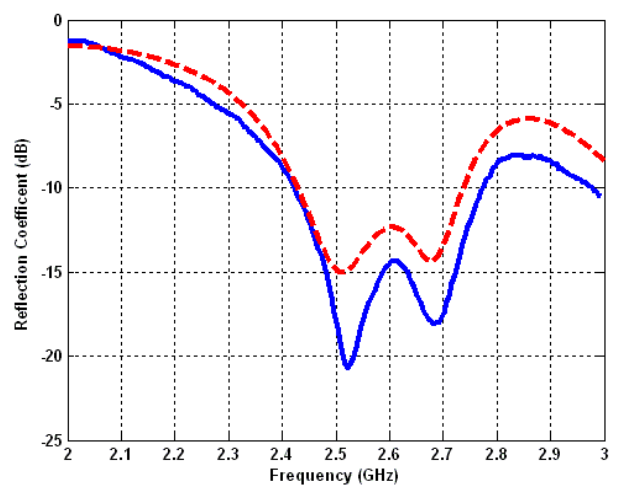

Fig. 7. Measured reflection coefficient $S_{11}$ for a 7-Element Dielectric Embedded SMTA, measured (solid line); simulated (dot line).

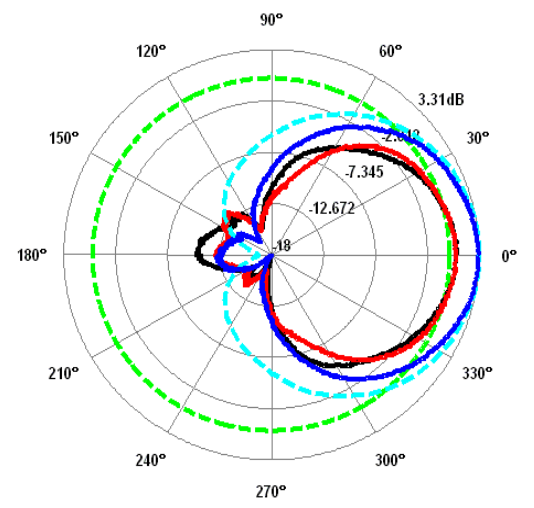

Fig. 8. Measured 2.4GHz H-Plane radiation pattern for a 7 Element Dielectric Embedded SMTA at beam pointing direction, $\theta=90^{\circ}$ (Blue); $\theta=60^{\circ}$ (Red); $\theta=130^{\circ}$ (Black); Simulated $\theta=90^{\circ}$ (Cyan --) ; -3dB Beamwidth (Green--).

The decision parameters in the structural optimization for this case study include the element heights, element array radius as well as the dielectric radius and height. To reduce optimization time, the element structural variables were kept within limits that were determined by available material and the overall size of the antenna.

\section{Results of Patch SMTA}

Figure 9 and Figure 10 illustrate simulated and measured reflection coefficients and radiation pattern of an optimized patch antenna array with seven circular elements (PASCE) and patch antenna array with seven hexagonal elements (PASHE), respectively, when the parasitic element No. 6 is open-circuited while the remaining short-circuited to ground. 


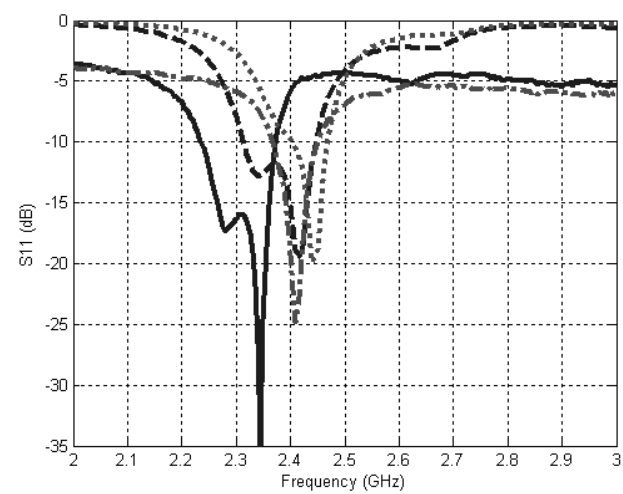

Fig.9 Simulated and measured reflection coefficients of PASHE and PASCE, where - Measured $\mathrm{S}_{11}$ of PASHE, - $-\mathbf{s}$ Simulated $\mathrm{S}_{11}$ of PASHE, $\boldsymbol{- \boldsymbol { n }}$ Measured $\mathrm{S}_{11}$ of PASCE and ...... Simulated $S_{11}$ of PASCE.

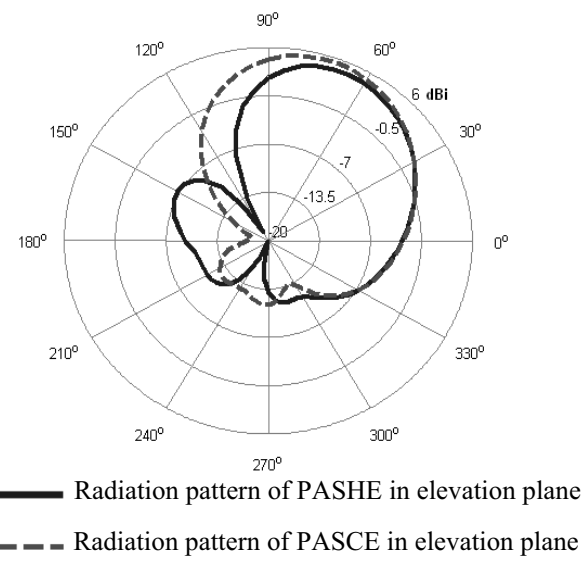

Fig.10. Simulated radiation pattern of PASHE and PASCE in elevation plane when the main beam lobe is steered in different directions in azimuth plane.

\section{Result comparison of SMTAs}

Table 1 presents the comparison of experiment results of SMTAs at the resonant frequency. The prototype SMTAs with four different structure configurations are shown in Fig. 11.

Table 1. Measured SMTA results

\begin{tabular}{||l|l|l||l||}
\hline \hline SMTA & $\begin{array}{l}\text { Reflection } \\
\text { coefficients } \\
\left(\mathrm{S}_{11}\right) \mathrm{dB}\end{array}$ & $\begin{array}{l}\text { Gain (max.) } \\
(\mathrm{dBi})\end{array}$ & $\begin{array}{l}\text { Frequency } \\
\text { Bandwidth } \\
\text { at -10dB }\end{array}$ \\
\hline Wire SMTA & -30 & $6 \mathrm{dBi}$ & $150 \mathrm{MHz}$ \\
\hline DE SMTA & -20 & $5.8 \mathrm{dBi}$ & $320 \mathrm{MHz}$ \\
\hline $\begin{array}{l}\text { Patch SMTA } \\
\text { (PASHE) }\end{array}$ & -35 & $4.90 \mathrm{dBi}$ & $140 \mathrm{MHz}$ \\
\hline $\begin{array}{l}\text { Patch SMTA } \\
\text { (PASCE ) }\end{array}$ & -25 & $5.44 \mathrm{dBi}$ & $85 \mathrm{MHz}$ \\
\hline
\end{tabular}

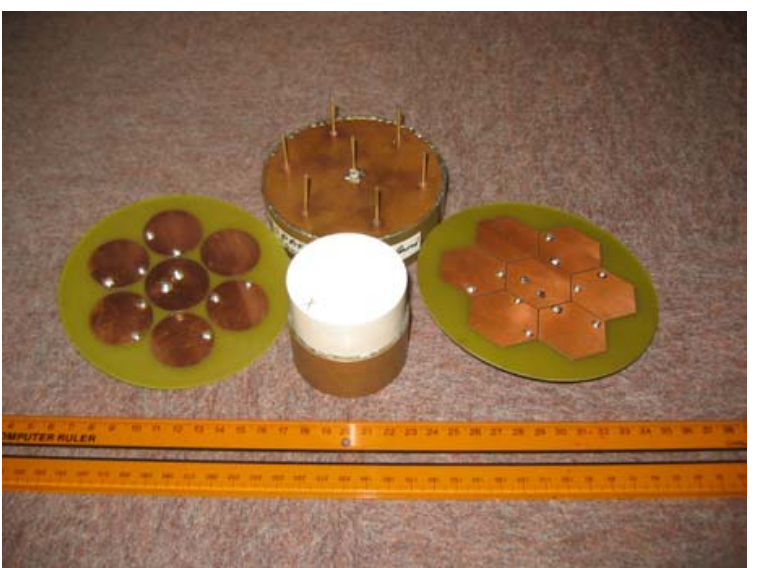

Fig.11. Prototype SMTAs with four different structure configurations.

\section{Conclusion}

The paper presented four different optimal designed low profile SMTA arrays. Wire SMTA and Patch SMTA can be used for Access Point (AP), while DE-SMTA is suitable for desktop or laptop computer in wireless network. The SMTA arrays with switched beam-forming circuit and algorithm can provide an ideal communication performance in the wireless communication and computing network, and further increase wireless security performances. Moreover, the performances of a low profile SMTA arrays can be improved significantly if some comprehensive multiobjective optimal methodology is implemented successfully, which is under the way of investigating of the authors.

\section{References}

[1] J. Sun, J. Lu and D. Ireland, "Increased Security Level using Space-division Approval in Wireless Computing Network," Proceedings of APMC-2005, vol. 3, 2005.

[2] R. Schlub, J. Lu and T. Ohira, "Seven element ground skirt monopole ESPAR antenna design from a genetic algorithm and the finite element method," IEEE Transactions on Antennas and Propagation, vol. 51, no. 11, pp. 3033-3039, Nov. 2003.

[3] J. Lu, D. Ireland and R. Schlub: Dielectric Embedded ESPAR Antenna for Wireless Communications Systems, IEEE Trans. on Antenna and Propagation, Vol. 52, No. 8, Aug. 2005, pp. 2437-2443.

[4] M. Shi, J. Lu and D. Ireland, "Smart Patch Antenna for Indoor Mobile Wireless Computing," Proceedings of APMC-2005, vol. 3, pp 1913-1916, Dec. 2005. 